\title{
Individual magnitude estimates for various distributions of signal intensity
}

\author{
DAVID M. GREEN, R. DUNCAN LUCE, and ALBERT F. SMITH \\ Harvard University, Cambridge, Massachusetts 02138
}

\begin{abstract}
Magnitude estimates of loudness were collected for several variations in the schedule of signal presentations. For wide ranges (about $50 \mathrm{~dB}$ centered at $65 \mathrm{~dB}$ ), the conditions were: random selection of 21 signals equally spaced in decibels, constrained selection so that each signal was used equally often but successive signals were always close together, constrained selection in which successive signals were always far apart, and random selection from a nonuniform distribution that consisted of two groups of equally spaced signals separated by a gap of $24 \mathrm{~dB}$. In addition, two other ranges, 10 and $30 \mathrm{~dB}$, were run with random selection of 21 equally spaced signals. The measures examined were: mean magnitude estimate as a function of signal intensity, coefficient of variation of the ratio of successive responses as a function of signal separation, and the correlation of the logarithm of successive responses as a function of signal separation. The basic question was whether all of the different schedules of signal presentation produce data that can be viewed as selections from appropriate regions of the 50-dB random signal selection data. To a degree, this was found, but with systematic exceptions.
\end{abstract}

In collecting sufficient individual magnitude estimates of loudness $(1,000-3,000$ responses) to permit estimates of the variability of the ratio of successive responses and also of their correlation (Green \& Luce, 1974; Green, Luce, \& Duncan, 1977; Luce \& Green, 1974, 1978), two highly patterned relations were found for most observers.

The first of these relations was that the size of the coefficient of variation (standard deviation divided by the mean) of the ratio of successive magnitude estimates depends sharply upon the difference in decibels between the corresponding signals. When the difference between successive signals is large, greater than 10-15 dB, the coefficient of variation is roughly constant, lying between .3 and 1 , depending upon the individual observer. When the signal difference is small, the coefficient of variation is smaller by a factor of some 3 to 5 . Put another way, a plot of the coefficient of variation of the ratio of successive responses vs. the decibel difference of the corresponding signals is a V-shaped function with its minimum near the 0 -dB signal difference and its maximum at signal changes larger than 10-15 dB.

The second relation, also dependent on the separation of the signals on successive trials, was the correlation between the logarithms of the successive responses. Correlations are computed between successive log responses for each distinct pair of signals, after which they are averaged over all pairs having the same decibel difference. A plot of this average correlation of successive log responses against the signal difference is an inverted V-shaped function, with a maximum average correlation between .70 and .95 occurring at about the $0-\mathrm{dB}$ difference. Low correlations, of zero or less, occur when the signal difference exceeds 20 to $30 \mathrm{~dB}$.

These phenomena are not restricted to loudness. The V-shaped function for the coefficient of variation was first noted by Mashhour (1964) for velocity, by Vlek and Beintema (1967) for area, and by Mashhour and Hosman (1968) for area and length; Baird, Green, and Luce (1980) have found it for judgments of area. The inverted V-shaped function for the correlation of log responses was first reported by Jesteadt, Luce, and Green (1977) and Green, Luce, and Duncan (1977), although the existence of sequential dependencies in magnitude estimation was noted earlier by Cross (1973) and Ward (1973). Ward (1979) has reported functions similar to ours.

To account for the systematic change in the coefficient of variation of response ratios, Green and Luce (1974) hypothesized an attention band, some 15 to $20 \mathrm{~dB}$ wide, which can be located anywhere on the intensity continuum. A signal falling inside the attention band is presumed to be better represented by virtue of a larger neural sample size than the same signal falling outside the band. If the band is located in the vicinity of the most recently presented signal, it follows that the coefficient of variation is smallest when the signal change is small and larger when the signal change is such that the current signal falls outside the band.

The similar dependence of the correlation of the logarithm of successive responses on difference of successive intensities suggests that the two results may be related, although the pattern of correlations does not appear to be due directly to differential sample sizes. The inverted-V shape for the corre- 
lation forces the coefficient of variation to exhibit some of the V-shaped pattern (Luce \& Green, 1978), but it does not appear to be a complete explanation, as can be shown by simulation of the attention band model.

These two relations have been observed in all our previous studies, except for one observer, who had a flat coefficient of variation and a flat ( $\sim .6)$ correlation function. These studies all used a large range of signal intensities-about $60 \mathrm{~dB}$-and random selection from a set of equally spaced signals. The attention band hypothesis leads us to expect that if the range were to be narrowed or, equally, if each signal was to be located within the band, then we should find that more and more of the data correspond to the apexes of these two functions. Equally, if signals were always placed outside the band, we should anticipate only a low or zero correlation and the larger coefficient of variation. Of course, existing evidence makes clear that signal range (Poulton, 1968; Teghtsoonian, 1971) and the distribution of signal intensities (Pollack, 1965) have some influence on the mean magnitude estimates.

Thus, both our attention-band notion and previous empirical evidence suggest that range and the distribution of successive signals might affect in systematic ways the coefficient of variation of successive response ratios and the correlation between successive responses as well as the mean response. Specifically, then, we manipulated the size and distribution of differences of signal intensity on successive trials in the following ways. For one group of observers, two different Markov chains were used to select signals so that asymptotically each signal was equally likely to occur, but successive signals were constrained in the one case to only small changes and in the other to only large ones. For both patterns of transitions, a 50-dB range was used. In three other conditions we used a random selection from equally spaced signals but three different ranges were used: 50, 30, and $10 \mathrm{~dB}$. For another group of observers, signals were selected from a distribution composed of two groups of signals, each including eight equally spaced signals over a 14-dB range, with the two groups separated by $24 \mathrm{~dB}$. Although the differences of successive signals did not occur with equal frequency, this condition involved both large and small, but not intermediate, changes in intensity from trial to trial.

The question was whether the results would appear to be consistent with the attention band hypothesis and, particularly, with the notion that the band is centered on the last signal.

\section{METHOD}

The signals, $1,000-\mathrm{Hz}$ tones of $500 \mathrm{msec}$ duration, were presented binaurally, in quiet, via TDH-39 headphones. The ob- servers were tested in single-wall sound-treated chambers (IAC-402A). Blocks of 100 trials were run with brief rests between blocks.

For four observers, signal sets spanning ranges of 50, 30, and $10 \mathrm{~dB}$ were used. Each set consisted of 21 signals equally spaced in decibels, centered at $65 \mathrm{~dB}$ SPL. In three conditions, successive signals were selected at random from one of these sets. In two other conditions, Markov transition matrices were used to program the selection of successive signals from the $50-\mathrm{dB}$ range signal set. Both transition matrices involved diagonal bands of five adjacent nonzero entries of probability $1 / 5$, with exceptions due to overflow at the end signals. For the small step case, the band was centered on the main diagonal, with an overflow occurring for the two signals at each end which received the entries $2 / 5,2 / 5,1 / 5$, and $2 / 5,1 / 5,1 / 5,1 / 5$, respectively. The matrix for large steps had the band centered at $\left(S_{n-1}, S_{n}\right)$ pairs $(1,11)$, $(2,12), \ldots(10,20),(11,21),(12,1),(13,2), \ldots(21,10)$, with overflow on the right placed on the extreme left. For example, with the band centered at $(10,20)$, the $1 / 5$ belonging to the nonexistent cell $(10,22)$ is placed in $(10,1)$. In each of the five conditions, the center intensity, $65 \mathrm{~dB}$, was presented for $10 \mathrm{sec}$ prior to each block of trials, and observers were instructed to call this 100 . All four observers participated in the same sequence of conditions: narrow range, wide-range/random, wide-range/small-step, wide-range/large-step, and intermediate range.

Five different observers were presented with signals chosen randomly from a set of 16 signals from a gapped 52-dB range; the signals were spaced $2 \mathrm{~dB}$ apart from 36-50 dB SPL and from 74-88 dB SPL. Two additional observers participated in the gapped and continuous wide-range conditions on alternating days.

The observers were instructed to assign numbers to the signals so that the ratio of the numbers assigned to successive tones was equal to the ratio of successive loudnesses. The observers were urged to keep the coefficient of variation of responses as small as possible. Following each run of 100 trials, they were informed both as to the value of the coefficient of variation averaged over all signals and the correlation between the logarithm of mean response and signal intensity. This latter statistic was monitored because one obvious way to achieve a low coefficient of variation is to use only a few responses. However, such a tactic lowers the correlation between stimulus intensity and mean response.

To acquaint observers with the magnitude estimation procedure, practice on approximately 1,500 trials was given on the narrowrange condition before the data reported here were collected.

Each trial began with a $500-\mathrm{msec}$ warning light on the response box. After a signal was presented, the observer responded by entering an integer between 1 and 99,999 on a response box. The number appeared on an LED display, and any mistake could be corrected. When satisfied with the response, the observer pressed a key which caused the response to be stored in the computer (PDP-15) and initiated the next trial. Approximately 2,100 observations were taken from each observer for each condition.

Observers were paid $\$ 3.50 / \mathrm{h}$ for their participation in the experiment.

\section{RESULTS}

\section{Magnitude Estimation Functions}

The mean magnitude estimates as a function of sound pressure level are shown in Figures 1, 2, and 3 for the three groups of observers. Although our main interest is not the shape of the psychophysical function, we comment briefly on the results. In Figure 1, two observers, N.P. and B.O., show magnitude estimation functions that appear to be essentially the same for all five experimental conditions. The other two 


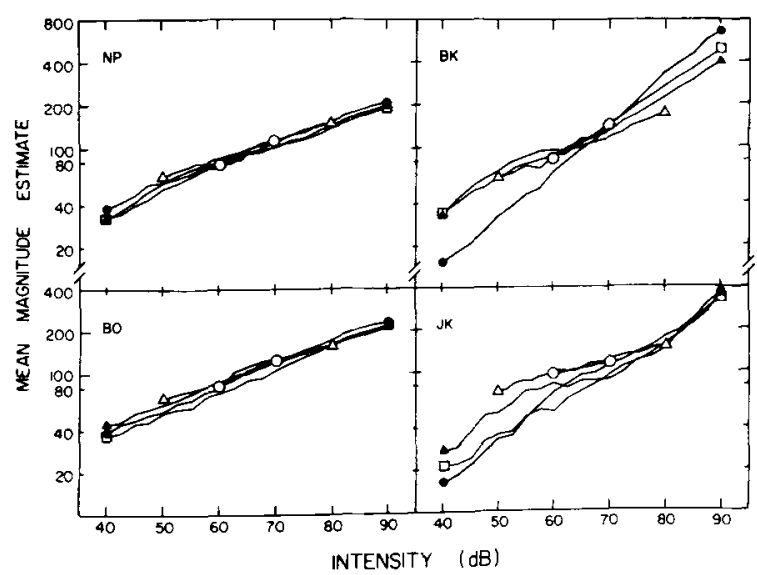

Figure 1. Mean magnitude estimates as a function of the sound pressure level of a $1,000-\mathrm{Hz}$ tone. Data from five distributions of intensity are presented for four observers. The open symbols represent the three ranges investigated: $10 \mathrm{~dB}$ (circles), $30 \mathrm{~dB}$ (triangles), and $50 \mathrm{~dB}$ (squares). The solid symbols are the data obtained from the experiment using a 50-dB range, but with successive stimuli restricted to small (circles) or large (triangles) changes in intensity.

observers, B.K. and J.K., exhibit a slight, but systematic, effect of stimulus transition probability on the shape of the magnitude estimation function. The largest slope occurs with small transitions. Random selection from equally spaced sets exhibits a slight, but systematic, decrease of the slope with range. This result is inconsistent with previous studies on the effect of range (Teghtsoonian, 1971). The general finding is that the slope of the magnitude estimation function increases as the physical range of the stimulus decreases. Of course, there are notable differences in procedure. Ours was a within-subjects design; the earlier work was between subjects. Ours involved many responses from each observer; the earlier work rested on many observers giving only a few responses to each signal. Ours involved a standard presented before each block of trials; the earlier work either involved no standard or repeated presentations of it. Any of these factors might underlie the discrepancy.

The data for the five observers who responded to the bimodally clustered signals (Figure 2) are, with the possible exception of those for S.L., as one would expect if psychophysical functions for a continuous signal range underlie the responses. In particular, there is no evidence that the observers "close the gap," as would occur if an attempt were made to maintain some prescribed distribution of responses. Surprisingly, the existence of the 24- $\mathrm{dB}$ gap is not at all obvious when listening to the signals: when checking the computer program for this condition, one of the authors thought the continuous distribution was being presented when, in fact, he was listening to the gap condition.

Figure 3 shows the data for two subjects who, on alternate days, were presented with either the continuous or the gapped set of intensities. It is clear that the gap produced little change in the magnitude

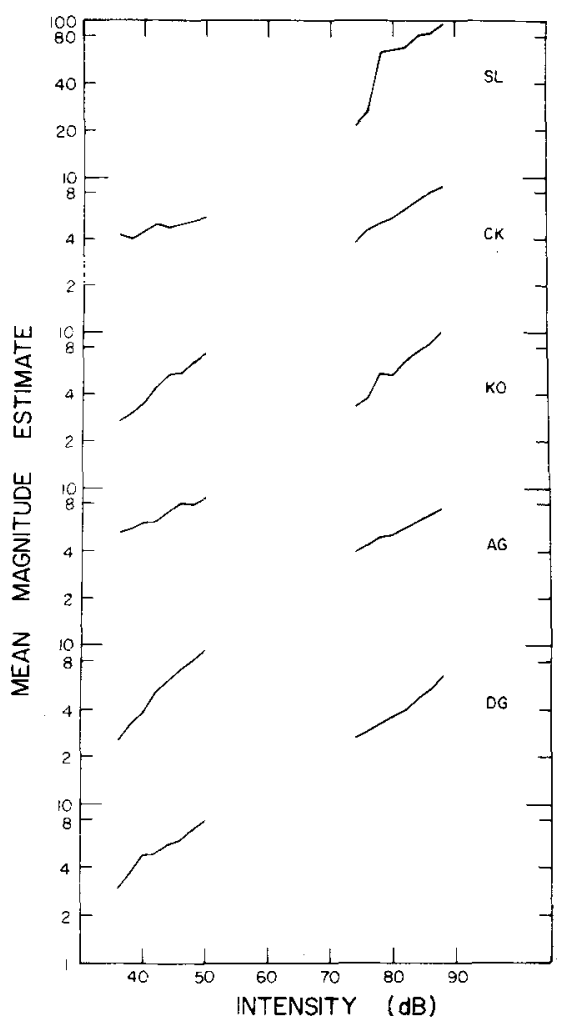

Figure 2. Mean magnitude estimates as a function of the sound pressure level of a 1,000-Hz tone for stimulus intensities in which there was a gap of $\mathbf{2 4} \mathrm{dB}$ in the middle of the range. Data from five observers are presented.

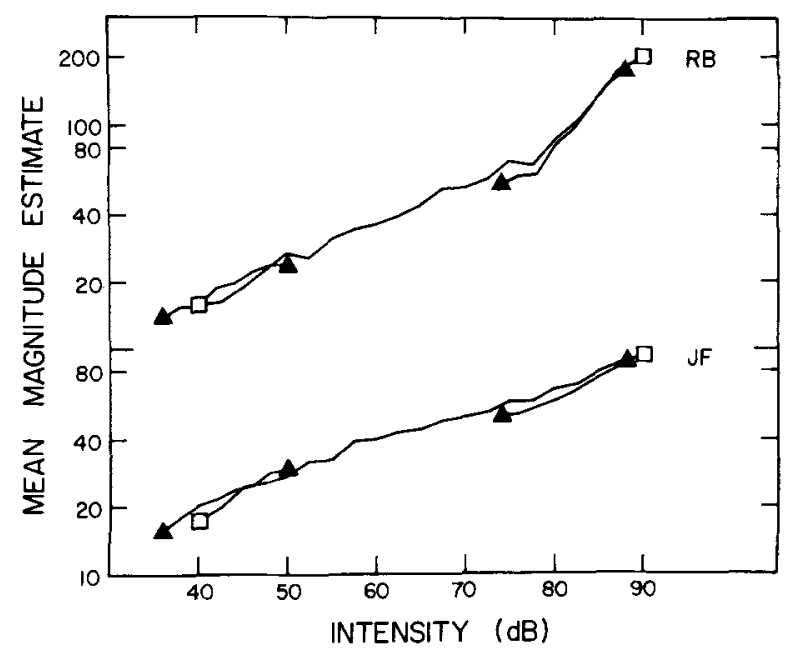

Figure 3. Mean magnitude estimates as a function of the sound pressure level of a $1,000-\mathrm{Hz}$ tone. Data are presented for two observers, who responded to a continuous (squares) and a gapped (triangles) stimulus distribution on alternate days. 


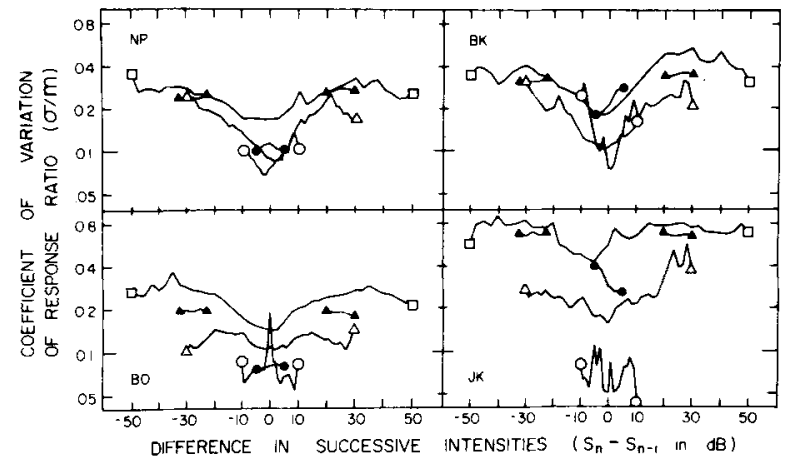

Figure 4. The coefficient of variation of the ratio of successive responses $(0 / \mathrm{m})$ as a function of the difference in sound pressure level on trials $n$ and $n-1$. Each point is based on all magnitude estimates for a given signal separation, regardless of the stimulus pair producing that ratio. Data from five experimental conditions are displayed in the figure; the symbol code is the same as that used in Figure 1. Data from four observers are displayed.

estimation function. When questioned, only one of the observers remarked that the distribution of stimuli was different from day to day.

\section{Coefficient of Variation of Response Ratios}

Figure 4 shows the coefficient of variation of the ratios of successive responses as a function of the decibel difference of successive signals for observers who estimated signals from the three intensity ranges and two special transition matrices. The $V$ pattern is apparent, and its minimum decreases with range. The observers were most variable, overall, for the 50-dB range. In the $50-\mathrm{dB}$ range data, the apex is smallest for observer B.O., about .15, and largest for observer J.K., about .43; larger changes in signal intensity result in coefficients of variation some two to three times greater than those produced by the smaller changes. The $\mathrm{V}$ shape is still evident in the 30-dB range data, except perhaps for observer B.O. The relative variability of response ratios for the 10-dB range shows practically no change with differences in intensity for two observers, J.K. and B.O., but a pronounced $V$ shape for B.K. and a less pronounced one for observer N.P. For both J.K. and B.O., the coefficient of variation for the 10-dB data is appreciably less than for either of the larger ranges.

The data obtained using nonuniform transition matrices are also shown in Figure 4. The coefficient of variation is some two to three times larger in the large-step condition than it is in the small-step condition and is approximately equal to or somewhat smaller than values for the comparable signal differences for the condition in which signal selection from the 50-dB range was random. The coefficients of variation for the small transitions are about the same as those obtained in the 10- $\mathrm{dB}$ range experiment for observers B.O. and N.P. and about the same as the corresponding part of the $50-\mathrm{dB}$ range function for observers B.K. and J.K.
Figure 5 presents the coefficient of variation of response ratios from the gap experiment, in which all changes are either small or large. For all of these observers, small signal changes produce smaller coefficients of variation than large changes. The coefficients of variation computed from the data of the two observers who alternated between the gapped and the continuous wide range are shown in Figure 6. The curves resemble two random samples of the same function.

\section{Correlation of Successive Log Responses}

Figure 7 shows the average weighted correlation coefficients of the logarithms of successive responses as a function of the decibel difference of the signal intensities on successive trials. (Recall, these correlations are computed separately for each distinct signal pair and only then averaged over those pairs having the same decibel difference.) The familiar inverted $V$ is the general pattern of these functions. For all observers, the functions for the 30 - and $50-\mathrm{dB}$ ranges are very similar. However, in the $10-\mathrm{dB}$ range condition, only for observer N.P. is the correlation uniformly high. For the other three observers, the 10-dB range yielded a sharply peaked function, with the correlation for observer J.K. actually dropping to near zero for the maximum changes of $10 \mathrm{~dB}$. When

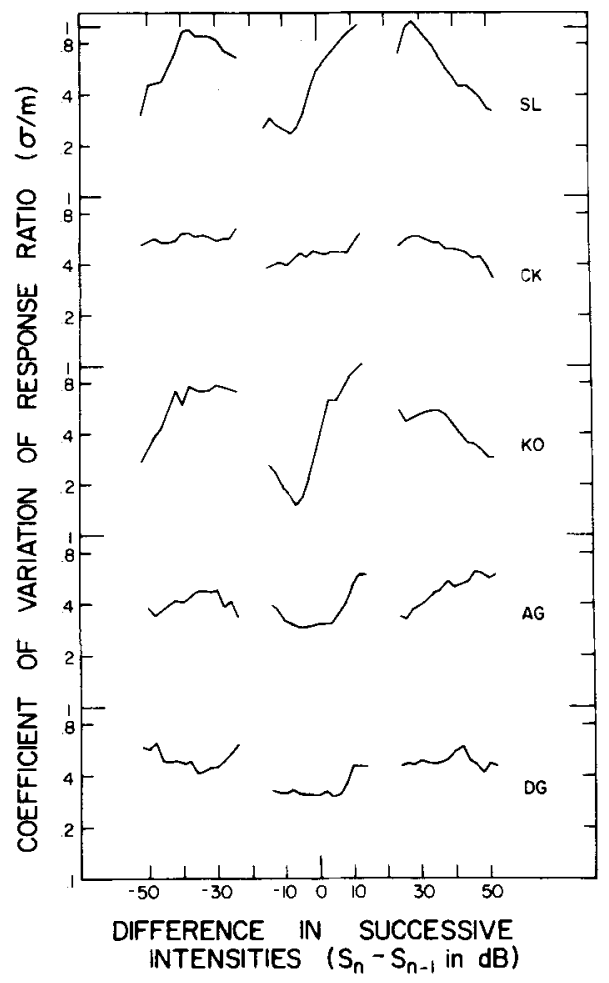

Figure 5. The coefficient of variation of the ratio of successive responses, as a function of the difference in sound pressure level on trials $n$ and $n-1$, for five observers who responded to signals from a gapped signal set. 


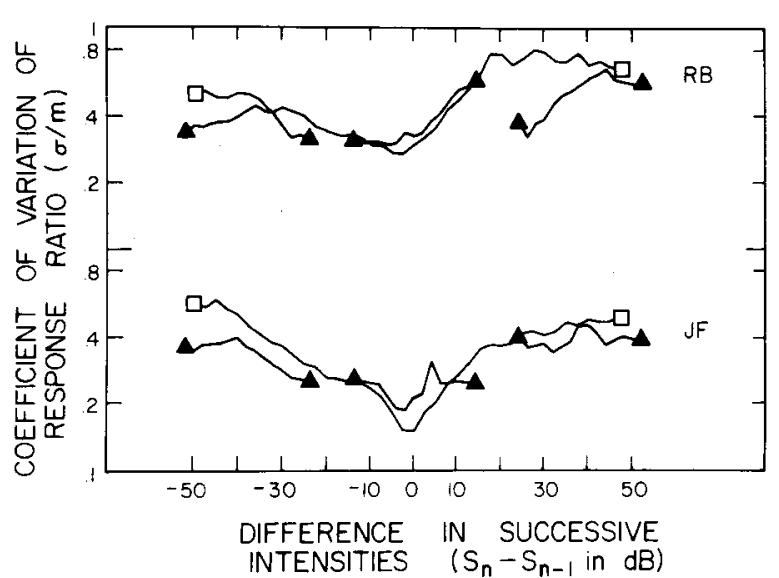

Figure 6. The coefficient of variation of the ratio of successive responses, as a function of the difference in sound pressure level on trials $n$ and $n-1$, for two observers who, on alternate days, responded to the continuous (squares) and gapped (triangles) signal sets.

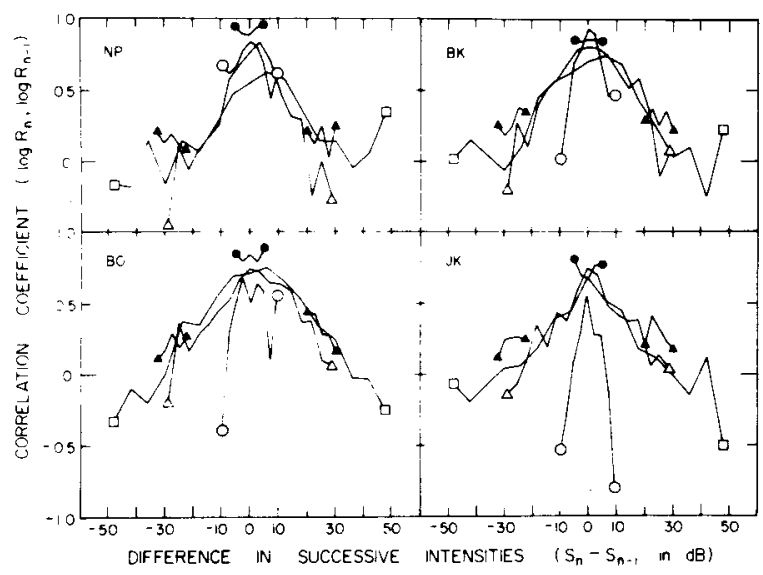

Figure 7. Coefficients of correlation between the logarithms of the responses on trials $n$ and $n-1$ as a function of the sound pressure level on the successive trials. A separate correlation is determined for each pair $\left(S_{n}, S_{n-1}\right)$ and then averaged across all the same stimulus separations. The results of five experimental conditions are presented. The symbol code is the same as that used in Figure 1. Data from four observers are displayed.

signal selection is constrained from trial to trial, the correlation of successive responses is approximately zero in the large-step condition and quite hightypically highest of all conditions-in the small-step condition.

The correlation data for the first gap experiment are shown in Figure 8. These, too, exhibit a sizable correlation for small changes-although not as high as we have observed elsewhere-and roughly zero correlations for large changes.

The correlation of successive responses of the two observers who alternated days on the gapped and the continuous wide-range signal sets are shown in Figure 9. Once more, the correlation data appear to be independent of stimulus distribution.

\section{DISCUSSION}

The attention band hypothesis has two features, one essential and the other not. The essential one is that the quality of the internal representation of a signal is better, because it is based on a larger sample size, when the signal is inside the attended region of

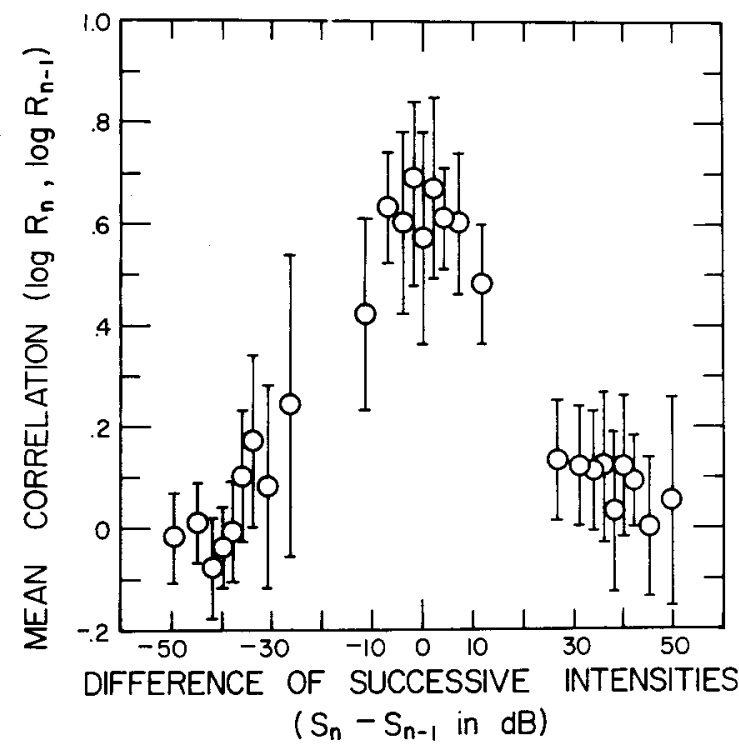

Figure 8. Coefficients of correlation between the logarithms of the responses on trials $n$ and $n-1$ as a function of the sound pressure level on successive trials for the experiment with a gap in the intensity range. The circles represent the mean correlations over five observers; the vertical bars are plus or minus one standard deviation based on the independent means.

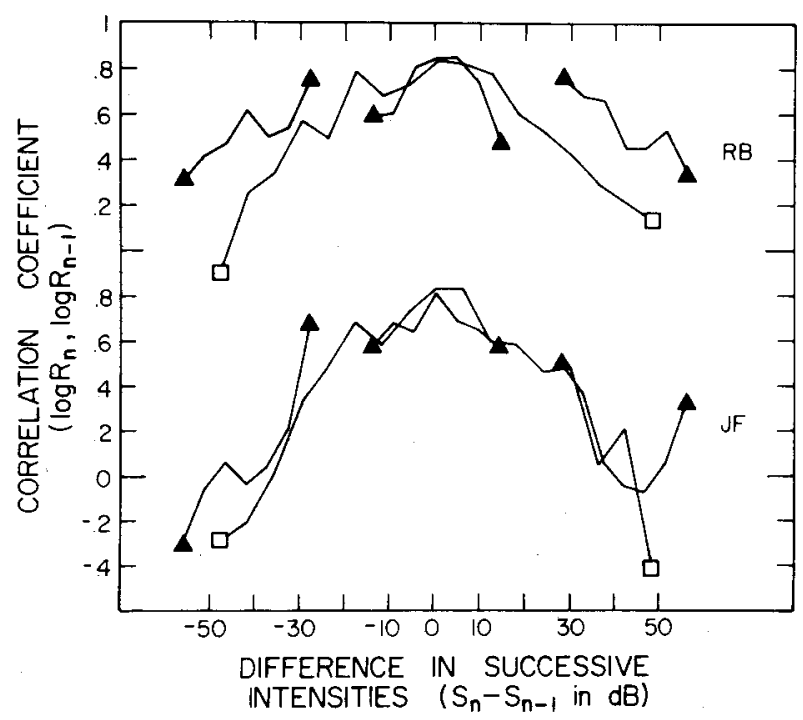

Figure 9. Coefficients of correlation between the logarithms of the responses on trials $n$ and $n-1$ as a function of the sound pressure level on successive trials. Values were determined as described in Figure 7. The results from two observers in two conditions are displayed; the symbol code is that used in Figure 3. 
intensity than when the signal lies outside the region of attention. The inessential one concerns the trial-bytrial adjustments of the location of the attention band, and we have been led to believe that the band tends to be centered in the region of the most recent signal. Operationally, this means that the separation between the current signal and the previous one correlates highly with the signal falling inside or outside the band. Clearly, it would be much better to gain full experimental control over its location, but so far experimental techniques to accomplish this have eluded us. Were the second hypothesis strictly correct, we should have found no effect on the mean, the coefficient of variation, or the correlation plots arising from modifications of the signal presentation schedule-whether it be the range of intensities, the distribution of signals over the range, or constraints on the intensity difference of successive signals. This would have meant a perfect (except for statistical fluctuations) superposition of all curves from the various conditions for each relationship.

What we have found is rather more complex.

For mean response as a function of signal level, superposition tended to be the case except for two observers who showed small effects of both range and sequential effects.

Range clearly affects the coefficients of variation of response ratios. All observers exhibited a decrease in this measure as the range was restricted. The coefficient of variation is also affected by effective range. When signals were selected from the 50-dB range under the constraint that all differences of successive intensities be small, observed values for three observers were less than, and for the fourth about equal to, those observed for equivalent signal differences with random signal selection. For two observers, these data and the 10-dB range data are substantially the same, whereas for two others the small-change data lie above the 10-dB data. In the gapped range condition in which signal differences were unpredictably either large or small, the effective range was presumably the same as the full range; the coefficient of variation curves for two observers in the gapped and continuous wide-range condition are comparable.

For the correlation of successive responses, the major deviation is that the correlations for the narrow range drop to zero for the extreme changes, whereas they are still quite large for the comparable position on the wide-range curve. In contrast to the coefficient of variation curves, the curves of correlation vs. intensity difference for the 30- and 50-dB ranges are practically superimposed. Moreover, the values for the small-step transition condition are strikingly high.

The conclusion is that the behavior is somewhat modified by changes in the presentation schedule, but that there is a considerable tendency for the main pattern to prevail. Since the deviations that do occur seem somewhat different for the coefficient of variation and the correlation, we suspect that two different, although perhaps partially related, phenomena are involved: It is clear that the naive attention-band account is insufficient, even for the coefficient of variation, and is quite inadequate to explain the correlation data.

The question of theoretical accounts that might be adequate to these and related data unfortunately seems to be complex, involving a moderate amount of mathematical derivation and parameter estimation. For that reason, we have elected to present those ideas in a separate theoretical paper.

\section{REFERENCES}

Baird, J. C., Green, D. M., \& Luce, R. D. Variability and sequential effects in cross-modality matching of area and loudness. Journal of Experimental Psychology: Human Perception and Performance, 1980, 6, 277-289.

Cross, D. V. Sequential dependencies and regression in psychophysical judgments. Perception \& Psychophysics, 1973, 14, 547 552.

GrEen, D. M., \& Luce, R. D. Variability of magnitude estimates: A timing theory analysis. Perception \& Psychophysics, 1974, 15, $291-300$.

Green, D. M., Luce, R. D., \& Duncan, J. E. Variability and sequential effects in magnitude production and estimation of auditory intensity. Perception \& Psychophysics, 1977, 22, 450 . 456.

Jesteadt, W., Luce, R. D., \& Green, D. M. Sequential effects in judgments of loudness. Journal of Experimental Psychology. Human Perception and Performance, 1977, 3, 92-104.

Luce, R. D., \& Green, D. M. The response ratio hypothesis for magnitude estimation. Journal of Mathematical Psychology, 1974, 11, 1-14.

Luce, R. D., \& GREen, D. M. Two tests of a neural attention hypothesis for auditory psychophysics. Perception \& Psychophysics, 1978, 23, 363-371.

Mashrour, M. Psychophysical relations in the perception of velocity. Stockholm: Almqvist and Wiksell, 1964.

Mashrour, M., \& Hosman, J. On the new "psychophysical law": A validation study. Perception \& Psychohysics, 1968, 3, 367-375

Poldack, I. Iterative techniques for unbiased rating scales. Quarterly Journal of Experimental Psychology, 1965, 17, 139-148.

Poulton, E. C. The new psychophysics: Six models for magnitude estimation. Psychological Bulletin, 1968, 69, 1-19.

TEghtsoonian, R. On the exponents in Stevens' law and the constant in Ekman's law. Psychological Review, 1971, 78, 71-80.

VLEK, C., \& BEINTEMA, K. Schaalaf-hankelykheid van psychophysisch gedrag [Scale-dependence of psychophysical behavior]. Hypothese, Tijdschrift voor de Psychologie (Leiden), 1967, 11, 111-125.

WAHD, L. M. Repeated magnitude estimations with a variable standard: Sequential effects and other properties. Perception \& Psychophysics, 1973, 13, 193-200.

WARD, L. M. Stimulus information and sequential dependencies in magnitude estimation and cross-modality matching. Journal of Experimental Psychology: Human Perception and Performance, 1979, 5, 444-459.

(Received for publication June 21, 1979; revision accepted March 14, 1980.) 